\title{
Design optimisation for an additively manufactured automotive component
}

\author{
Meisam Abdi* \\ Faculty of Engineering, \\ University of Nottingham, \\ Nottingham, UK \\ and \\ Faculty of Technology, \\ De Montfort University, \\ Leicester, UK \\ Email: meisam.abdi@dmu.ac.uk \\ *Corresponding author
}

\section{Ian Ashcroft and Ricky D. Wildman}

Faculty of Engineering, University of Nottingham, Nottingham, UK

Email: Ian.Ashcroft@nottingham.ac.uk

Email: Ricky.Wildman@nottingham.ac.uk

\begin{abstract}
The aim of this paper is to investigate the design optimisation and additive manufacture of automotive components. A titanium brake pedal processed through selective laser melting (SLM) is considered as a test case. Different design optimisation techniques have been employed including topology optimisation and lattice structure design. Rather than using a conventional topology optimisation method, a recently developed topology optimisation method called Iso-XFEM is used in this work. This method is capable of generating high resolution topology optimised solutions using isolines/isosurfaces of a structural performance criterion and extended finite element method (XFEM). Lattice structure design is the other technique used in this work for the design of the brake pedal. The idea is to increase the stability of the brake pedal to random loads applied to the foot pad area of the pedal. The use of lattice structures can also significantly reduce the high residual stress induced during the SLM process. The results suggest that the integration of the design optimisation techniques with a metal additive manufacturing process enables development of a promising tool for producing lightweight energy efficient automotive components.
\end{abstract}

Keywords: topology optimisation; lattice structures; additive manufacturing; automotive; powertrain; extended finite element method; XFEM; selective laser melting; SLM.

Reference to this paper should be made as follows: Abdi, M., Ashcroft, I. and Wildman, R.D. (2018) 'Design optimisation for an additively manufactured automotive component', Int. J. Powertrains, Vol. 7, Nos. 1/2/3, pp.142-161. 
Biographical notes: Meisam Abdi is currently an Early Career Academic Fellow at the Faculty of Technology, De Montfort University, UK. He received his $\mathrm{PhD}$ in Mechanical and Manufacturing Engineering from the University of Nottingham in 2015. His research is focused on structural optimisation techniques and also design and optimisation strategies for additively manufactured components.

Ian Ashcroft is a Professor of Solid Mechanics at the University of Nottingham and a member of the Additive Manufacturing Research Group. His expertise is in both experimental and computational mechanics. Since 2005, he has focused his research on the application of solid mechanics to additive manufacturing, particularly in developing multi-physics modelling techniques for AM processes and the post processing performance of parts and the development of design and optimisation techniques to exploit the design freedoms of various AM technologies.

Ricky D. Wildman is a Professor of Multiphase Flow at the University of Nottingham. He is interested in understanding the mechanics relevant to the deposition and formation of material within additive manufacturing processes, and how computational methods can be deployed to understand and assist.

This paper is a revised and expanded version of a paper entitled 'Design optimisation for additive manufacture of lightweight automotive components' presented at 3rd Biennial International Conference on Powertrain Modelling and Control (PMC 2016), Loughborough University, 7-9th September 2016.

\section{Introduction}

Within the last few decades, emergent needs such as energy efficiency, environmental protection and refinement in the automotive and aerospace sectors have been pushing back the boundaries of current state of art architecture in order to meet future emissions and performance criteria. Recently developed structural optimisation techniques as well as advanced manufacturing processes are the main key tools for providing a platform toward multifunctional lightweight energy efficient systems.

Recently, there has been a growing interest in the use of additive manufacturing (AM) for building lightweight powertrain and automotive components (Cooper et al. 2015). The key advantage of AM is that it offers a significantly greater design freedom than that in conventional manufacturing processes, which allows the built part to be closer to the optimised design (Bracket et al., 2011). Therefore, AM aims to enable the manufacturing of geometrically complex features which are not possible/significantly difficult to manufacture using traditional manufacturing processes. Examples of these geometrically complex structures include lattice structures and topologically optimised structures. These lightweight structures are mainly used to deliver a specified mechanical performance such as stiffness and impact absorption.

The aim of topology optimisation is to achieve the best possible design for a structure by systematically changing the material distribution within a specified design domain of the structure toward an optimal solution. The general approach for topology optimisation 
includes an iterative process of numerical analysis of design domain, for instance finite element analysis (FEA), followed by a sensitivity analysis and an update process of design variables. There have been different types of methods proposed for topology optimisation of structures, operating based on material distribution variation. Examples include solid isotropic material with penalisation (SIMP) (Bendsøe, 1989; Zhou and Rozvany, 1991), and bidirectional evolutionary structural optimisation (BESO) (Querin et al., 1998; Huang and Xie, 2010). Both these methods are element-based, which means a finite element or a property of a finite element, for instance density in SIMP, is considered as a design variable. As a result, the boundary of the final design obtained through these methods is not clearly defined as it is represented by finite elements. Therefore, the solution from topology optimisation will require further interpretation and post processing, such as smoothing and shape optimisation in order to become a manufacturable design (Hsu et al., 2001; Victoria et al., 2009). This is further discussed in the next section followed by examples of SIMP and BESO solutions. OptiStruct (Altair Engineering Inc.) is an example of software designed to enable the SIMP method of topology optimisation to be applied to real components. Other software such as Nastran (MSC Software) and Abaqus FEA (Dassault Systèmes) also have option to apply similar density-based approaches to find the solution to topology optimisation problems. As the final solutions are represented by element relative densities, there is a need to threshold the densities at an arbitrary value to get a discrete solution. This could reduce the optimality of the solutions while the post-processing is still required to get a manufacturable solution. In this study, a recently developed method called Iso-XFEM (Abdi et al., 2014a, 2014b) is used for topology optimisation. This method aims to achieve high resolution topology optimised solutions which require no more/only a little post processing before manufacturing.

The second element of the design process used in this study is lattice structure design. Lattice structures have potential to deliver high levels of stiffness and energy absorption while, offering a significant reduction in part weight. Another interesting property of lattice structures is that compared to topology optimised solutions, lattice structures are more robust to problems with multiple objectives or those that include uncertainty in the loading conditions (Maskery et al., 2015).

In this study, the two design elements mentioned above are employed for design optimisation of a brake pedal. The main objective of the study is to investigate the feasibility of the application of these techniques to design optimisation of an automotive component manufactured by AM. The brake pedal were produced in Ti-6Al-4V alloy using SLM (an AM process used to produce three-dimensional metal parts by fusing fine metal powders together using a laser beam). Following this general introduction, the next section of the paper introduces the topology optimisation method used in this study. The sections after investigate the practical challenges associated with the design optimisation and additive manufacture of the component.

\section{Topology optimisation}

Topology optimisation is expected to find the best material layout for a structure through finding the number and location of holes and the connectivity of structural members within the design space. Thanks to FEA, the complex topology optimisation problem can be simplified to finding the properties of finite elements, assuming each element or a 
property associated to that element as a design variable of the optimisation problem. In SIMP, design variables are element relative densities. Through an iterative optimisation process, design variables are updated using a gradient based optimisation method such as optimality criteria method. Due to the continuous nature of the design variables, the resulting solution can be represented not only with solid and void regions, but with intermediate densities. However, as these are not generally manufacturable, a power-law approach is used to move the intermediate elemental densities $(0<\rho<1)$ towards a $0 / 1$ solution by penalising the intermediate densities (Sigmund, 2001). Figure 1(a) shows a cantilever under a point load at the tip. Figure 1(b) shows the stiffest design obtained for the cantilever using SIMP method for a target volume of $50 \%$ of the design space. It can be seen that the boundaries are represented with finite elements, and as previously mentioned, there is a need to threshold the densities at an arbitrary value to get a discrete representation of the boundary.

BESO is an alternative material distribution method which operates by systematically removing and adding elements (rather than changing the densities as in SIMP) until it reaches convergence. The method is based on the assumption that the optimised design can be achieved by gradually removing inefficient material, e.g. the material in low stress regions of the design space, and adding the material to most efficient regions, e.g. high stress regions of the design space. Therefore it allows achieving a final solution with binary (0/1) representation. However, as can be seen from the BESO solution of the cantilever beam in Figure 1(c), the final solution is still represented with elements, requiring further post processing to generate a smooth manufacturable topology.

Figure 1 Topology optimisation solutions achieved using different methods implementing the same FE mesh (see online version for colours)

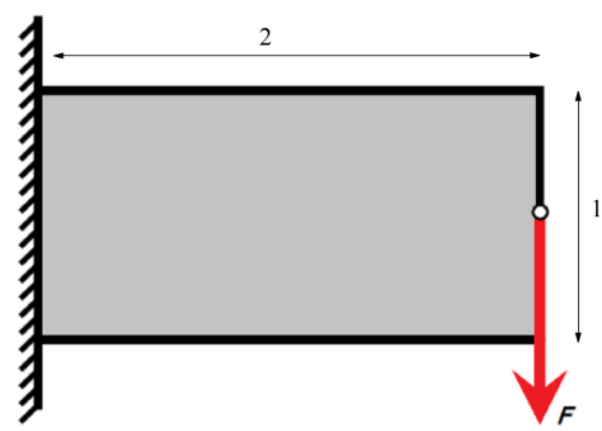

(a)

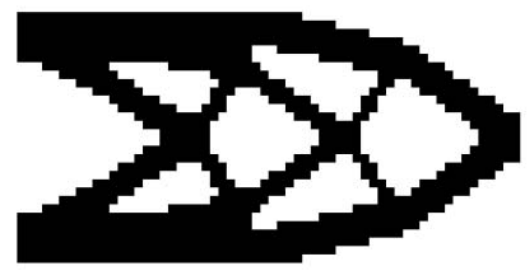

(c)

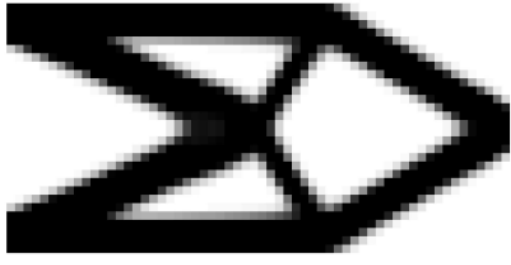

(b)

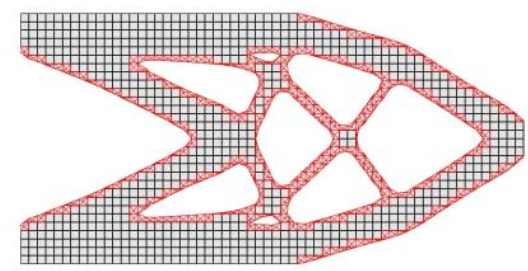

(d)

Notes: (a) Design domain; (b) SIMP solution; (c) BESO solution; (d) Iso-XFEM solution. 
Figure 2 (a) Illustration of isoline approach for representing the design boundaries where the intersection of SP distribution (SED in here) with MLP defines the current state of the boundary (b) Implicit representation of a 2D design space and the structure's geometry using relative structural performance (c) Design space decomposed into solid region $(\alpha(x)>0)$, void region $(\alpha(x)<0)$ and boundary $(\alpha(x)=0)$ (see online version for colours)

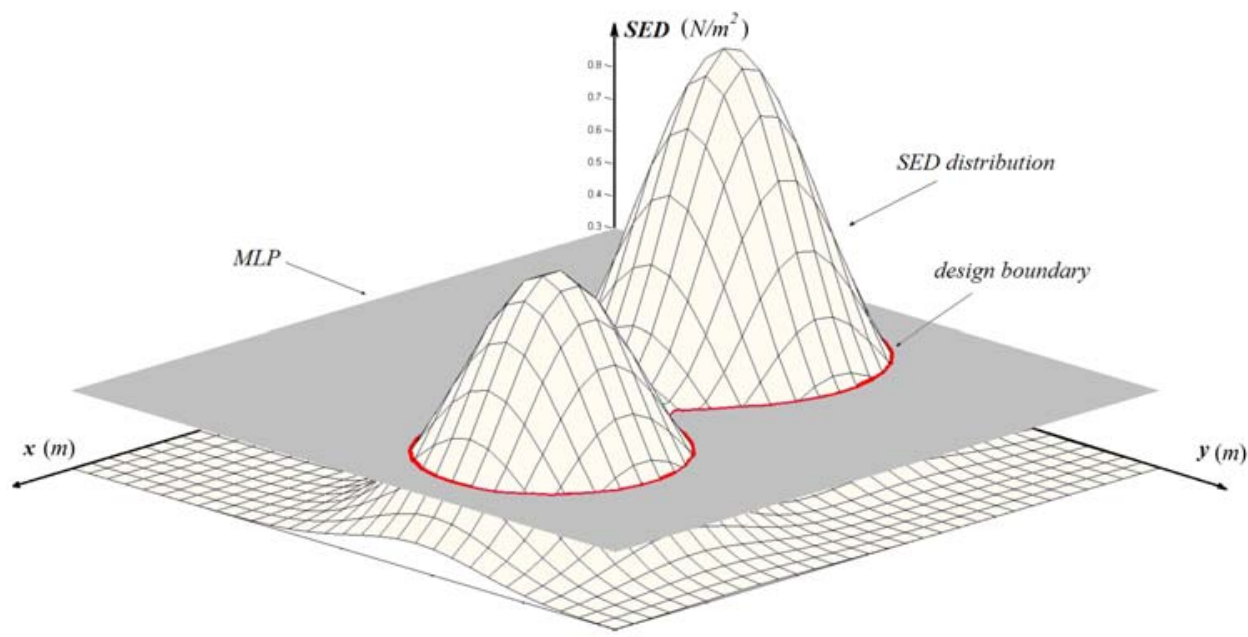

(a)

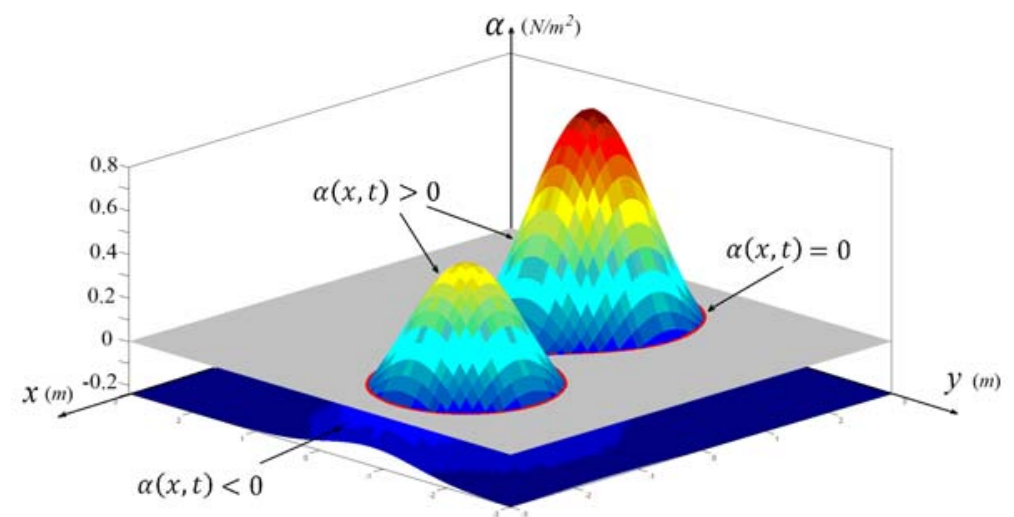

(b)

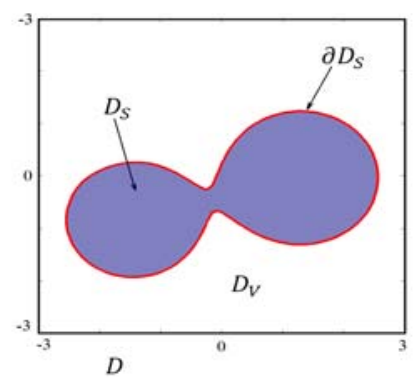

(c) 
The method used in this study, called Iso-XFEM, is an evolutionary optimisation method, similar to BESO, however, it doesn't suffer from the limitations of the conventional element based methods of topology optimisation in terms of boundary representation. The method operates by gradually removing and redistributing material within the elements of the design domain (rather than removing/adding elements as in BESO). This is achieved by representing the boundaries of the structure using contours of a structural performance criterion, such as von Mises stress, and calculating the properties of the finite elements which lie on the design boundary using extended finite element method (XFEM). An evolutionary optimisation algorithm is also integrated with these to enable a smooth material removal process. Figure 1(d) shows Iso-XFEM solution of the cantilever problem of Figure 1(a). It can be seen that unlike the two previous solutions, the solution achieved using the Iso-XFEM method is represented with clearly defined smooth boundaries. The three different elements of the Iso-XFEM method are explained in the next sections.

\subsection{Isoline/isosurface scheme for structural optimisation}

Isolines and isosurfaces define points of a constant value of a function in $2 \mathrm{D}$ and $3 \mathrm{D}$ spaces, respectively. The aim of isoline/isosurface design is to represent the boundaries of the structure using contours of a structural performance (SP) criterion such as von-Mises stress or strain energy density (SED). In order to realise this design approach in a structural optimisation problem, the design boundaries can be defined by the intersection of the structural performance distribution with a minimum level of performance (MLP) identified in each optimisation iteration (Victoria et al., 2009; Abdi et al., 2014b), as illustrated in Figure 2(a). Based on a relative performance value which is given by

$$
\alpha=\mathrm{SP}-\mathrm{MLP} \text {, }
$$

this scheme allows partitioning the design domain $(D)$ into solid $\left(D_{S}\right)$ and void $\left(D_{V}\right)$ regions, as

$$
\alpha(x): \begin{cases}>0 & \text { solid phase }\left(D_{S}\right) \\ =0 & \text { boundary }\left(\partial D_{S}\right) \\ <0 & \text { void phase }\left(D_{V}\right)\end{cases}
$$

Figures 2(b) and 2(c) illustrate how the scheme is used to partition the design space shown in Figure 2(a) into solid and void regions.

\subsection{Extended finite element method}

The isoline/isosurface scheme discussed in the previous section allows superimposing the design boundary on a fixed grid finite element mesh. By implementing this scheme, the finite element design space is divided into three classes of elements including solid elements, void elements, and boundary elements, i.e., the elements which lie on the design boundary having both solid and void subdomains. Using the classical finite element method, the contribution of solid and void elements to the structure's stiffness can be evaluated by assigning solid and void (very weak) material properties to those elements, respectively. However, in the case of boundary elements, classical FEA 
requires computationally expensive remeshing operations along with local refinement near the boundary to generate purely solid and void elements in that region. An alternative approach for calculating the properties of the elements near the boundary is the use of extended finite element method (XFEM) which doesn't need remeshing. The XFEM approximation space for modelling holes and inclusions, i.e., material/void interface as exists in topology optimisation, is given by (Sukumar, 2001):

$$
u(x)=\sum_{i} N_{i}(x) H(x) u_{i}
$$

where $N_{i}(x)$ are the classical shape functions associated to the nodal degrees of freedom, $u_{i}$. The value of the Heaviside function $H(x)$ is equal to 1 for the nodes and regions in the solid part of the design and switches to zero for nodes and regions in the void part of the design domain.

This XFEM scheme for modeling the material/void interface in topology optimisation can be realised by dividing the solid domain of the boundary elements into sub-triangles [in 2D problems as shown in Figure 3(a)] or sub-tetrahedra [in 3D problems as shown in figure 3(b)], and then performing numerical integration over the solid triangles/tetrahedra using Gauss quadrature method (Abdi et al, 2014b).

Figure 3 XFEM interation scheme (see online version for colours)

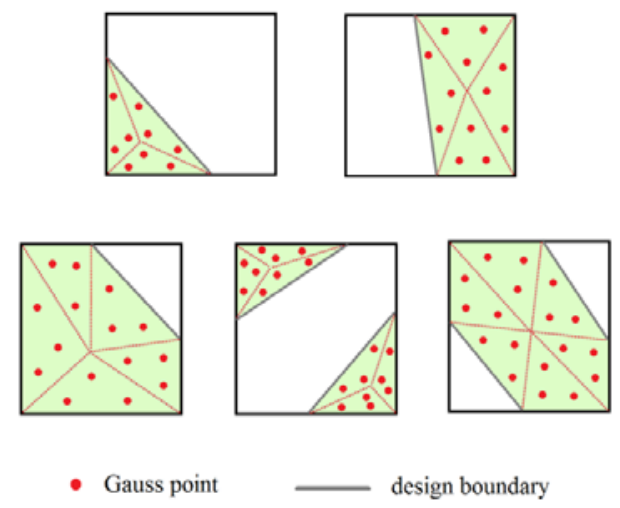

(a)

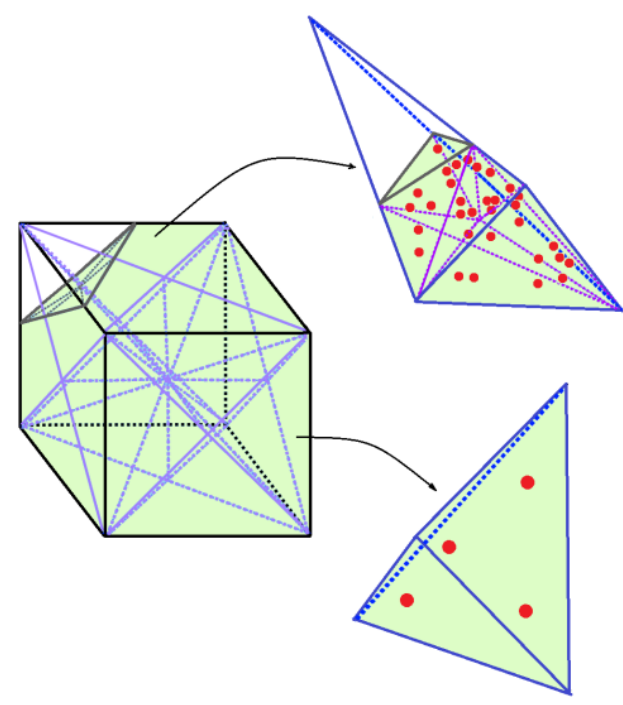

(b)

Notes: (a) Solid domains of 2D boundary elements are divided into sub-triangles;

(b) Solid domains of 3D boundary elements are divided into sub-tetrahedra.

\subsection{Evolutionary-based optimisation method}

The optimisation algorithm used in this study is based the on the simple assumption that the structure evolves toward an optimised topology and shape by gradually removing the inefficient material from its design domain. This is the same assumption used in evolutionary structural optimisation (ESO) method, however instead of removing (or 
adding as in BESO) the material in an elemental level, the optimisation operates at a global level of structural performance by the use of isoline/isosurface design approach. Therefore, the material can be removed/added within the finite elements. In order to characterise the efficiency of material usage in the design domain, an appropriate structural performance criterion is selected. Material is then removed from low relative performance regions $(\mathrm{x} ; \alpha(x)<0)$ and redistributed to the high relative performance regions $(\mathrm{x} ; \alpha(x)<0)$. In every optimisation iteration, the target volume of the design is calculated before any region is added to or removed from the structure. The target volume of the design which typically decreases through the evolutionary optimisation process for the current iteration is given by

$$
V_{i t}=\max \left(V_{i t-1}(1-E R), V^{c}\right)
$$

where $E R$ is the volume evolution rate and $V^{c}$ is the specified volume constraint. After finding the target volume for the current iteration, the MLP associated with that target volume is calculated. This could be achieved through an iterative process, for instance by defining upper and lower bands for MLP (which are equal to the maximum and minimum SP in first iteration, respectively), finding the volumes corresponding to the upper and lower bands, averaging and updating the upper and lower bands until the difference between the volumes corresponding to the upper and lower bands is smaller than a minimum value.

\section{Design optimisation of a brake pedal}

The method was considered for optimising a brake pedal with the loads and boundary conditions shown in Figure 4(a). The aim was to support the design and development of an additively manufactured brake pedal for formula student race car program in De Montfort University. An existing design for the pedal is shown in Figure 4(b).

The objective of the optimisation problem was to maximise the stiffness (minimise the compliance) of the pedal subject to a volume constrain. Two designs are explored in this section:

1 an optimised brake pedal with a volume of $15 \%$ of the initial design domain $(\mathrm{VF}=0.15)$ which is approximately the same volume as that in the existing brake pedal

2 an optimised brake pedal with a volume of $10 \%$ of the initial design domain $(\mathrm{VF}=0.10)$. The objective function compliance is given by

$$
C=\frac{1}{2} U^{T} K U=\frac{1}{2} F^{T} U
$$

where $U, K$ and $F$ are the global displacement vector, global stiffness matrix and global force vector in FE model, respectively.

The compliance can be minimised by removing material from low SED regions of the design space (Huang and Xie, 2010). Therefore, SED is used as the structural performance (SP) criterion for maximising the stiffness of the pedal using the Iso-XFEM method. Elemental values of SED can be calculated from 


$$
S E D_{e}=\frac{1}{2} u_{e}^{T} k_{e} u_{e} / v_{e}
$$

where $u_{e}$ and $k_{e}$ are the element's displacement vector and stiffness matrix, respectively, and $v_{e}$ is the element volume.

Figure 4 (a) Design domain and boundary conditions of the brake pedal (b) An existing design for the brake pedal (see online version for colours)

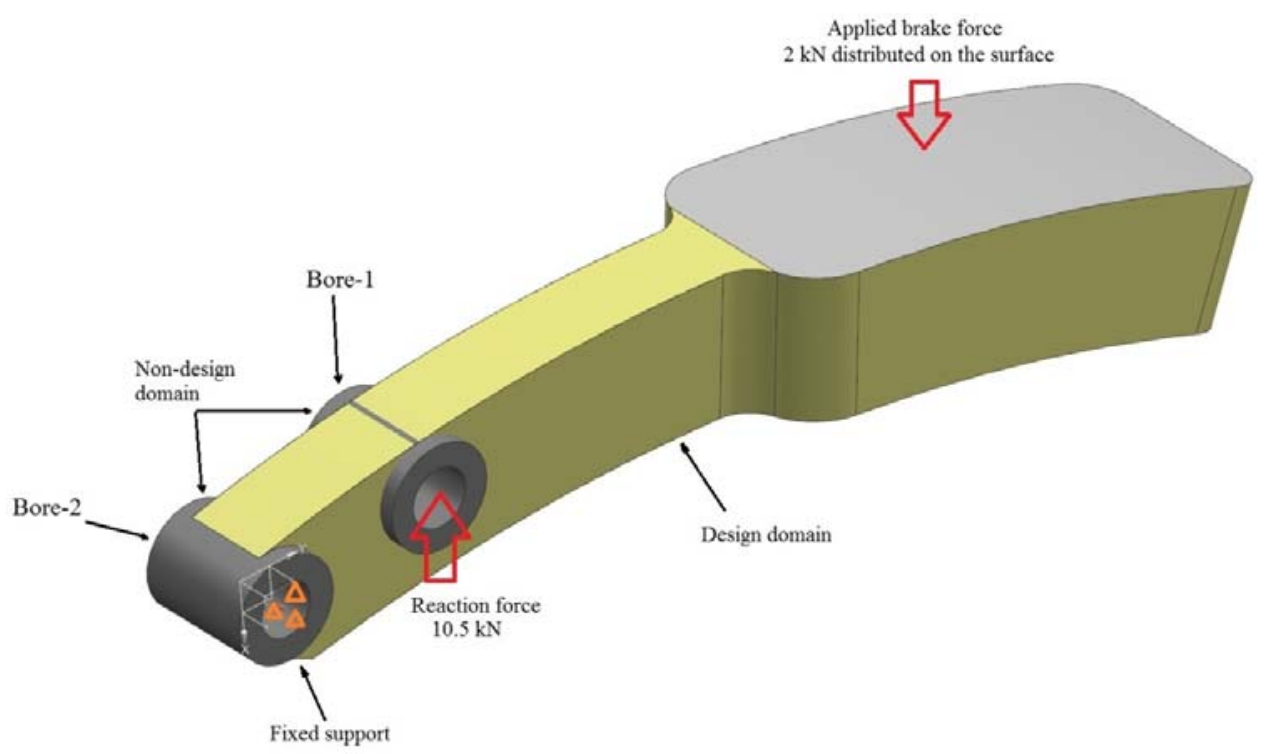

(a)

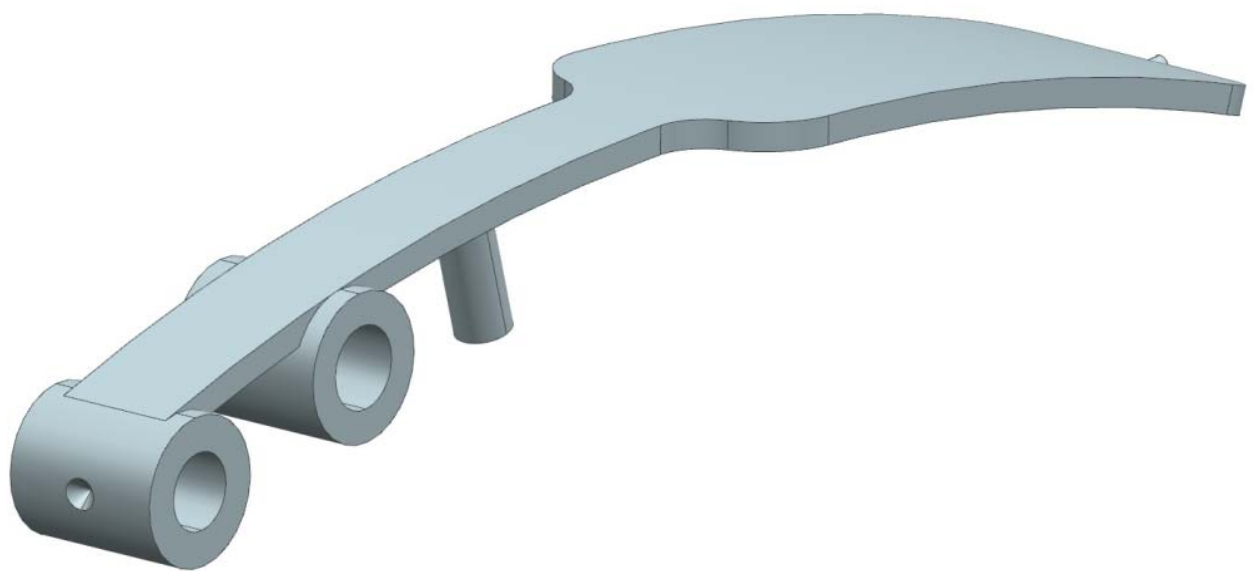

(b)

For simplicity of the finite element modelling, the $2 \mathrm{kN}$ brake force shown in Figure 4(a) was assumed to be uniformly distributed on the top surface of the pad. In reality, there will be some variation in exactly how the load is applied in practice dependent on the driver. This issue is addressed in section 5 of the paper by amending the design in the 
foot pad area of the pedal. A cylindrical reaction bar is fitted into bore-1 during installation of the brake pedal. Assuming free rotation around the axis of bore-2, a reaction force of $10.5 \mathrm{kN}$ results from the $2 \mathrm{kN}$ brake force. As the resultant moment produced by the two forces around the axis of bore- 2 is zero (no reaction moment), all FE nodes on the internal surface of bore- 2 were fixed for simplicity and stability of FEA.

Figure 5 Topology optimisation of the brake pedal component for target volume fraction of $\mathrm{VF}=0.15$ : (a) evolution histories of objective function (SE) and volume fraction (VF)

(b) Iso-XFEM solution (half of the pedal) (see online version for colours)

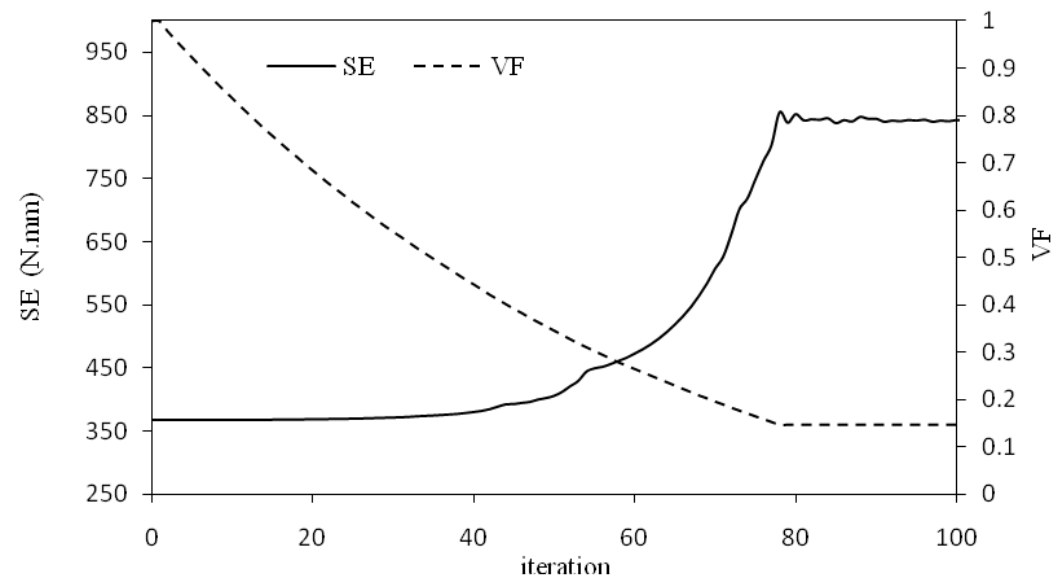

(a)

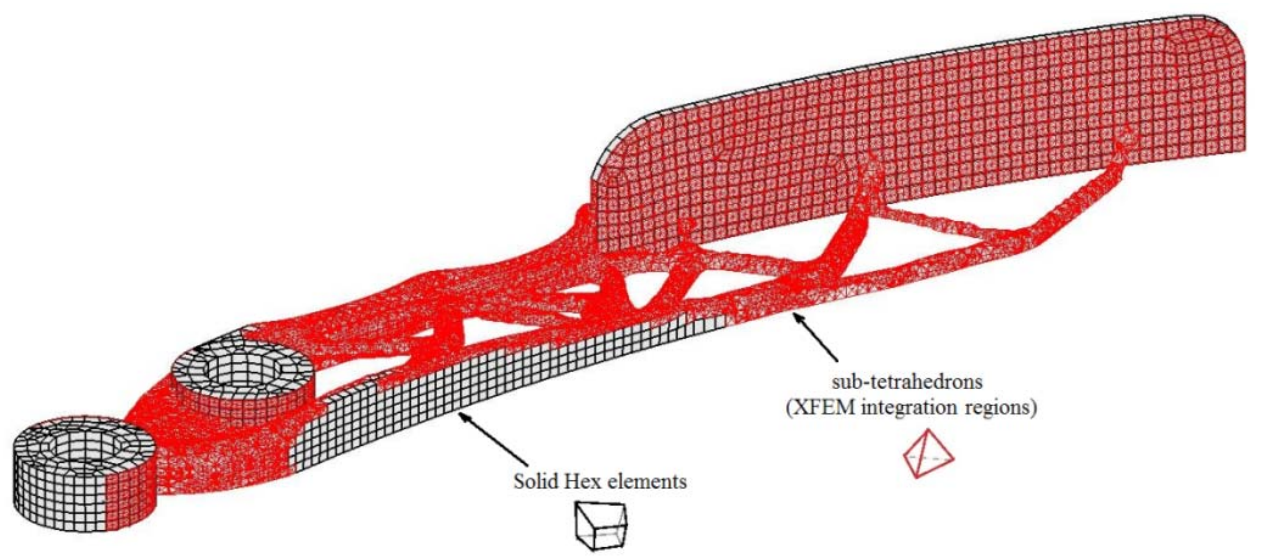

(b)

An initial FE mesh was generated using the commercial FEA software Altair Hypermesh. Due to the symmetry of the problem, only half of the structure was considered for the analysis using approximately 16000 hexahedral elements. The mesh was imported into MATLAB to apply the Iso-XFEM optimisation method. A volume evolution rate of ER = 0.02 was used in the evolutionary optimisation process. The material properties of selectively laser melted Ti-6Al-4V (Simonelli, 2014) were used in the optimisation: Young's modulus of $\mathrm{E}=113 \mathrm{GPa}$ and Poisson's ration of $v=0.33$. The solution for the 
first design $(\mathrm{VF}=0.15)$ converged after 90 evolutionary iterations as shown in Figure 5(a). Figure 5(b) shows the Iso-XFEM solution for the brake pedal for VF $=0.15$. It can be seen that despite using a coarse starting mesh, a relatively smooth solution has been achieved. As the surface of the solution is represented by triangles, it can be easily translated to sterolithography (STL) file format which is the de facto file format for manufacture in the AM sector. Figure 6(a) shows the optimised design for $\mathrm{VF}=0.15$ after inclusion of all non-design regions fallowed by a few iterations of smoothing. Similarly, the second design was obtained from the application of the method to the optimisation of the brake pedal with a target volume of $10 \%$ of the initial design space $(\mathrm{VF}=0.10)$ as can be seen in Figure 6(b).

Figure 6 Final optimised designs for the brake pedal after inclusion of all non-design regions (a) design for target volume fraction of $\mathrm{VF}=0.15$, and (b) design for target volume fraction of $\mathrm{VF}=0.10$ (see online version for colours)

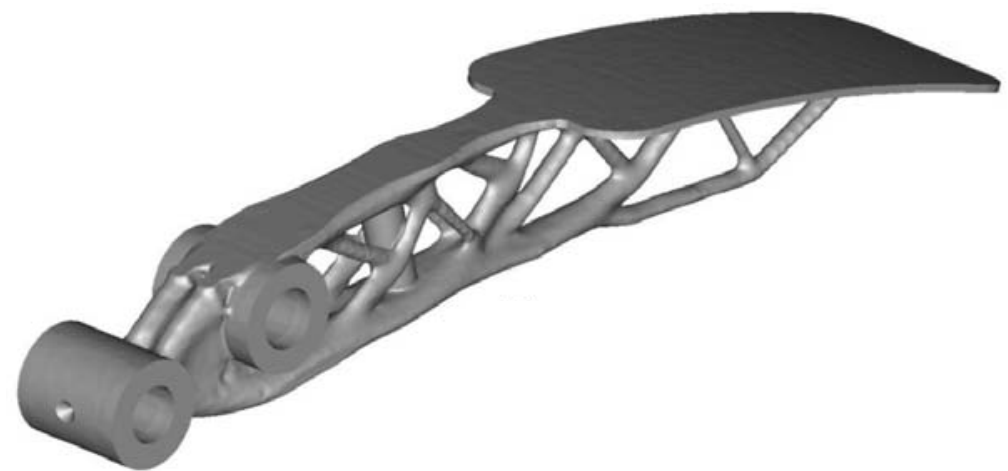

(a)

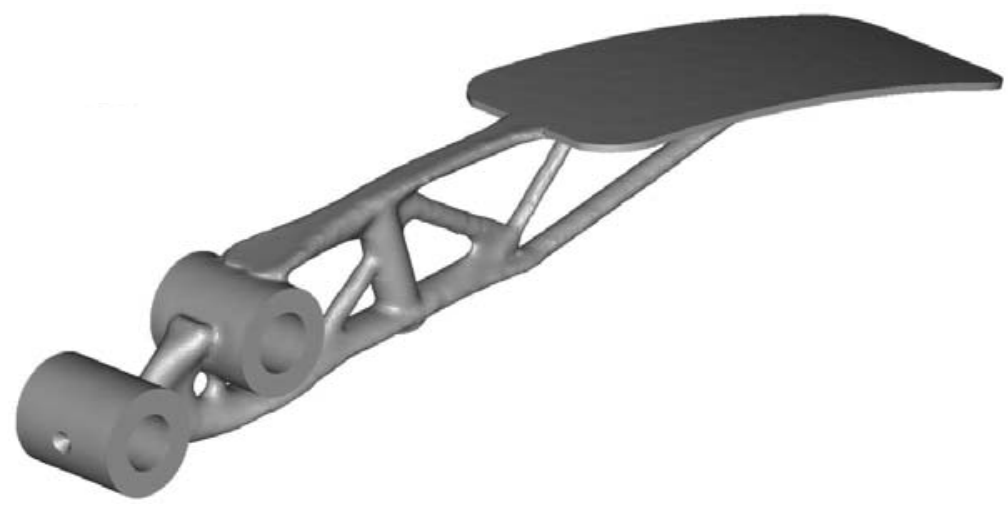

(b)

\subsection{FEA of the different designs}

The two optimised designs were compared with the existing design of the pedal in terms of their stiffness and the equivalent von-Mises stresses. A very fine structured hexahedral mesh was used to generate the FE model of the three considered designs. The commercial 
FEA package MSC Marc was used to solve the linear system of FE equations. Figure (7) shows the contours of von-Mises stress for the three designs. It can be seen that the two optimised designs [Figures 7(b) and 7(c)] have much lower levels of von-Mises stress than the existing design [Figure 7(a)]. It can also be seen that the distribution of vonMises stress is more uniform in the two optimised solutions than that in the existing design.

Figure 7 Contours of von-Mises stress for the three investigated designs: (a) the existing brake pedal, (b) the optimised design for $\mathrm{VF}=0.15$ and (c) the optimised design for $\mathrm{VF}=0.10($ see online version for colours $)$

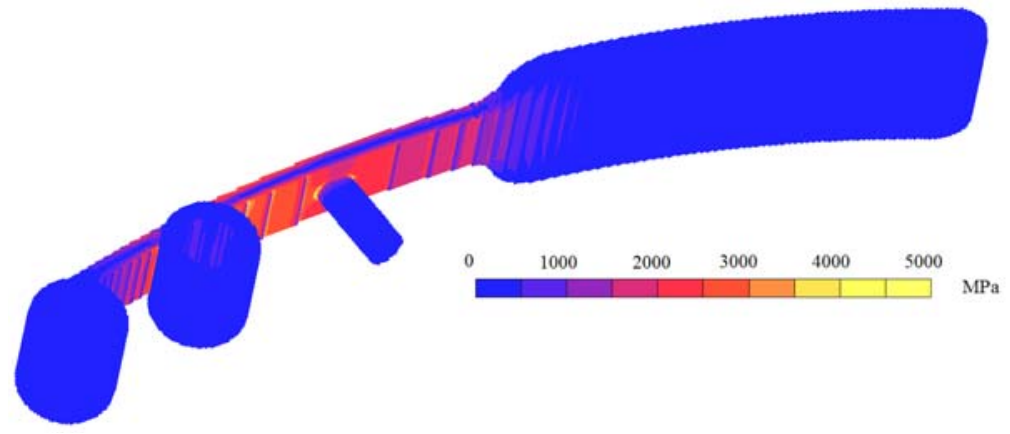

(a)

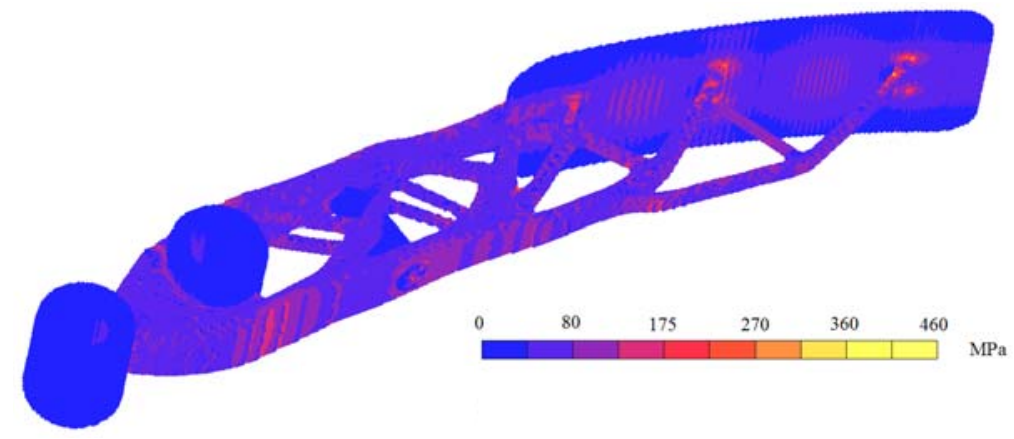

(b)

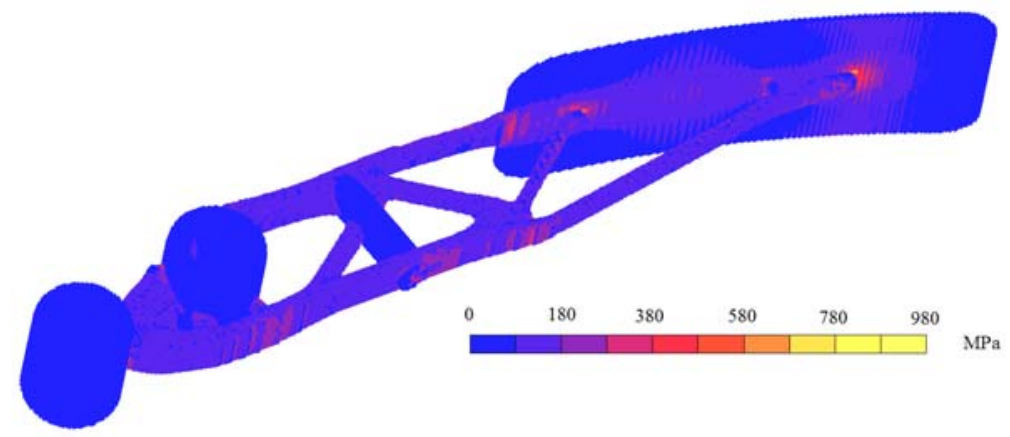

(c) 
Table 1 compares the objective (compliance) values of the three designs as well as the maximum von-Mises stresses achieved through the FEA. It can be seen that even with smaller design volumes, the two optimised designs have significantly smaller compliance values (i.e., much larger values of stiffness) than the existing design. This can be attributed to the fact that unlike the two optimised designs, the existing pedal doesn't have any structural members below the pad area to support the pad against deflection. Similarly the maximum von-Mises stresses of the two optimised designs are much smaller than that of the existing pedal. Although the existing pedal has less geometrical complexity than the two topology optimised solutions, the increased performance as well as the reduced weight of the optimised solutions suggest that they are the preferred designs for additive manufacture.

Between the two optimised designs explored in this section, the design optimised for $\mathrm{VF}=0.15$ was selected in this study for AM. As can be seen from Table 1 , the volume of this design is slightly smaller than that of the existing pedal, and the maximum von-Mises stress seen in this design is smaller than those in the other two designs. Also the topology optimised design for $\mathrm{VF}=0.15$ has high levels of geometrical complexity, which is suitable for our case study.

Table 1 Comparison of the three designs for the brake pedal in terms of their design volume, maximum von-Mises stress and compliance

\begin{tabular}{lccc}
\hline & Volume $\left(\mathrm{cm}^{3}\right)$ & Max von-Mises stress $(\mathrm{MPa})$ & Compliance $(\mathrm{N.m})$ \\
\hline Existing pedal & 72.1 & 5012 & 95.312 \\
Optimised design $(\mathrm{VF}=0.15)$ & 70.8 & 458 & 0.994 \\
Optimised design $(\mathrm{VF}=0.10)$ & 54.6 & 976 & 2.032 \\
\hline
\end{tabular}

\subsection{Further remarks on the application of the method to automotive and powertrain components}

The optimisation strategy used in this study was to minimise compliance subject to a target volume fraction. We didn't consider a maximum stress constraint in the optimisation problem, however the von-Mises stresses calculated for the optimised solutions were below the yield stress of the material used (selectively laser melted Ti-6Al-4V). Minimising compliance subject to a target volume has been commonly used for topology optimisation since the calculation of sensitivities is straightforward in this case. An alternative definition of the optimisation problem can be to minimise the volume of the structure subject to a specified compliance and/or a maximum von-Mises stress. Also, if any region of the final design violate the maximum von-Mises stress limit (for instance a region of high stress concentration), there is a need for further shape optimisation and reanalysis to reduce the stresses in that region.

The proposed optimisation method can be implemented for the design of further automotive, including powertrain, components. Examples of powertrain components which can be targeted using this method are transmission housings and powertrain mounting brackets. To date, there have been few published studies on the application of topology optimisation techniques to the design of powertrain components. Wu et al. (2016) used Altair Optistruct software, which utilises a density-based (SIMP) approach for topology optimisation, to optimise the stiffness of an engine bracket. Kandreegula et al. (2015) also used Optistruct to investigate stiffness optimisation of a transmission 
housing. In the case of powertrain components under dynamic loading condition, the objective should include the optimisation of both stiffness and natural frequencies. A mounting bracket subjected to both static and dynamic loadings was designed by Zhao et al. (2015) through a multi-objective topology optimisation approach implemented using the Optistruct software.

Unlike the above mentioned studies which used the commercial Optistruct software to generate optimised designs, the results of this study have been achieved by implementing the Iso-XFEM method programmed in MATLAB, without the use of commercial structural optimisation software. The aim was to investigate the applicability of the method on the design and optimisation of automotive/powertrain components. The main advantage of the proposed method compared to conventional density based methods is the fact that it enables the generation of high resolution designs which need little postprocessing before manufacturing. In terms of time efficiency, the Iso-XFEM method has a slightly higher computational cost than the conventional density based methods (dependent on the number of elements used for the FEA). However, the higher computational time cost for the topology optimisation can be offset by the benefit of the reduced post-processing requirement of the Iso-XFEM solutions. Further discussion regarding the time efficiency of the method can be found in Abdi et al. (2014a).

Depending on the nature of loading, further considerations may be required for the application of the method to real powertrain components. There might be a need to take into account fatigue effects, thermal effects, frictional behaviours and other NVH issues, for instance to constrain the location of the centre of gravity for rotatory components.

\section{Additive manufacture}

In this study, selective laser melting (SLM) process was used to manufacture the Titanium brake pedal. The process starts by slicing a 3D CAD model, usually defined by standard STL file format, into a number of layers, creating a 2D image of each layer. For each layer, a laser scan path is defined. Each layer is then sequentially recreated by depositing powder layers, one on top of the other, and melting their surface using a laser beam.

Despite significant design freedom that SLM process offers, there are a few manufacturing issues which should be taken into account to ensure fabrication success. These include generation of high residual stress during the process and support structure requirement. The SLM process involves a rapid heating and melting of material which is followed by a rapid solidification. The induced thermal variations cause the areas of the scanned layer to expand/contract at different rates resulting in the generation of a high residual stress which can cause the component to distort (Vora et al., 2015). To prevent deformation of the part and process failure, support structures made from the same material as the SLM component are fused to the substrate plate and various locations across the laser melted component, holding geometries in place. Large overhanging features of the component built parallel to the powder bed are typical areas requiring the most support. Although oversupporting a component can help to prevent distortion, it will be difficult to remove the support structure from the part and from the base plate after the build. Therefore it is important to find the best build orientation to minimise the support structure requirement. 
In this work, Renishaw AM250 machine was used to manufacture the topology optimised brake pedal. Marcam AutoFab package was used to add support structures to the designed component and to slice the $3 \mathrm{D}$ model before sending it to the machine for manufacturing. In order to reduce the residual stress effects and use less support structures, the component was printed on its side as shown in Figure 8. However, it can be seen from Figure 8 that the first try for printing the component was not very successful since the foot pad area of the pedal lifted off the build platform, causing the stud at the end to break off. Distortion is a common issue in large Titanium parts made with SLM due to the very high induced residual stresses (Qian et al., 2016). It is well understood that increasing the size of parts produced with SLM can intensify the residual stress effects/part distortion (Papadakis et al., 2014). In this case, it was not possible to add more support structures to the overhang surface of the foot pad as the section was too thin. Increasing the thickness of the solid pad could have resulted in increased residual stress/distortion in that section (due to the increase in mass and the heat absorbed during the SLM process), while increasing the overall weight of the component. The next section presents how this issue was addressed by introducing porosity inside the pad using lattice structure design techniques.

Figure 8 Brake pedal manufactured using SLM process (see online version for colours)

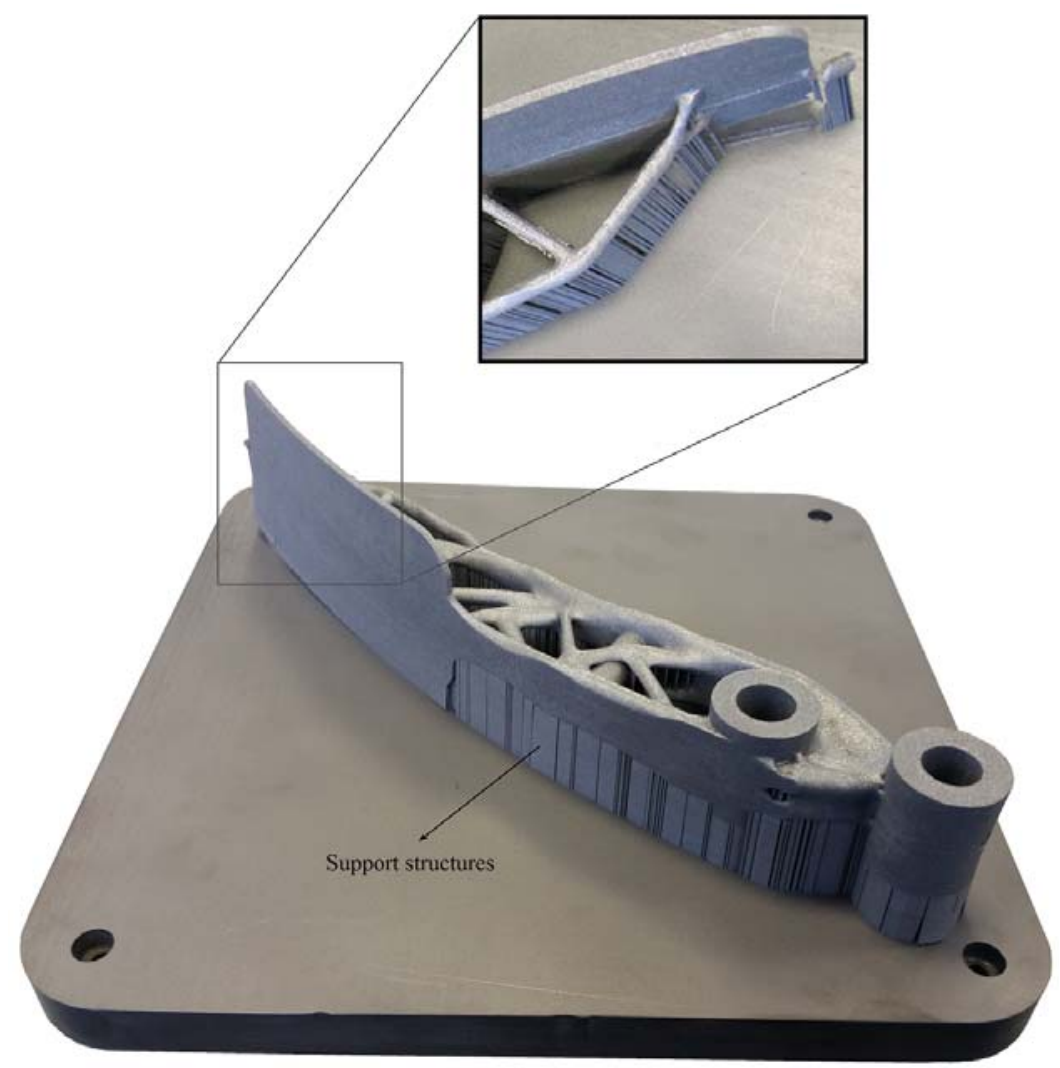

Note: A large distortion was observed in the foot pad area of the pedal. 


\section{Lattice design}

In the previous sections of the paper, design optimisation and AM process for manufacturing the brake pedal were presented. However there are a few technical difficulties regarding the design and manufacturing of this component. From design point of view, the foot pad is under a $2 \mathrm{kN}$ load applied by the driver. The optimisation solution obtained in Section 3 was based on the assumption that the load is uniformly distributed on the surface of the foot pad. However, dependent on the size of the driver, whether they are braking conventionally, left foot or heal toe, the position of the load will change on the surface of the foot pad. In order to consider the effect of the load position, one may need to define separate load cases for this problem and modify the objective function of the optimisation problem based on the number, magnitude and the probability of the load cases. This could make the optimisation problem much more complex. From manufacturing point of view, as mentioned in the previous section, a high residual stress was observed in the build resulting the foot pad lifted off the build platform, while it was not possible to add more support to the overhang surface of the thin foot pad. These two issues were the motivation to use a different technique, lattice structure design, to address these difficulties.

Figure 9 (a) Strut-based (BCC, BCCZ , FCC and PFCC) and TPMS (Gyroid network phase and matrix phase) unit cells (Aremu et al., 2014) (b) A structure filled with Gyroid lattice cells

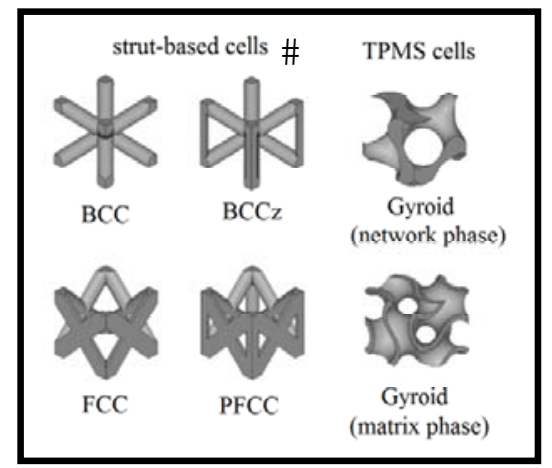

(a)

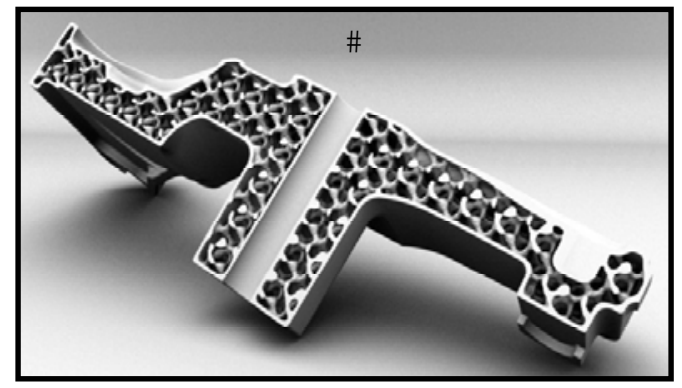

(b) 
Lattice structures are the structures filled with repeating units (cells). There are a range of different cell types offering different mechanical and thermal properties. Figure 9(a) shows a number of different lattice unit cells classified as strut-based cells and triply parodic minimal surface (TPMS) cells. The mechanical properties of the cells are highly dependent to the cell type, cell size and cell density (Aremu et al., 2014). In SLM, in particular we are interested in self-supporting lattice unit cells i.e. the cells that do not include overhanging features which would require the addition of supporting structures (Maskery et al., 2015). Therefore, the self-supporting cells can be used inside a structure as internal functional members of a structure [Figure 9(b)], as well as outside a structure as support structure for the SLM process (Hussein et al., 2013).

In addition to their enhanced mechanical properties, lattice structures offer robust solutions to problems which include uncertainty in loading conditions. This is the case in the design optimisation of the brake pedal as the position of the applied load by the driver can change on the foot pad area of the pedal. Another property of lattice structures when manufactured through SLM process is that a much less induced residual stress is expected during the build compared to a bulk volume of the same amount of material, simply because less heat energy is absorbed by a cellular structure compared to a solid structure during the SLM process. Therefore, cellular structures have the potential to reduce the overall residual stress as well as support structure requirement for the AM part if they are appropriately embedded into the component.

In this study in order to increase the robustness of the foot pad to random loads, and also reduce the residual stress induced to the foot pad area of the pedal, the thickness of the pad was increased from $2 \mathrm{~mm}$ to $4 \mathrm{~mm}$, and the internal region of the pad was filled with body centred cubic (BCC) lattice of density 0.2 . The BCC unit cell was chosen as it is a self-supporting cell. Therefore, the lattice structure can work as support structure for internal region of the pad, while it remains with the component after the build as structural members of the part. BCC lattice also has a high mechanical isotropy in $x, y$ and $z$ loading directions, suitable for problems which include uncertainty in loading conditions. A solid skin of thickness $1 \mathrm{~mm}$ was used on the surface of the foot pad as can be seen in Figure 10. Four small holes near the four corners of the bottom of the pad were designed to enable removal of the loose powder after the build (can be seen in Figure 11). By increasing the thickness of the lattice pad, it was possible to add more support structures to support the overhang surface of the pad, thus reducing the chance of component distortion and process failure. Due to the use of low density lattice structure inside the pad, the increase in the thickness of the pad didn't have a significant effect on the part weight. The overall design volume increased from $70.8 \mathrm{~cm}^{3}$ (the optimised design with a solid pad of thickness $2 \mathrm{~mm}$ ) to $72.9 \mathrm{~cm}^{3}$ (the optimised design with a lattice pad of thickness $4 \mathrm{~mm}$ ) which is close to the volume of the original design (72.1 $\mathrm{cm}^{3}$ ). However, the optimised design benefits from a much higher stiffness and lower levels of von-Mises stress compared to the original design of the pedal.

The final manufactured component after removing the external support structures is shown in Figure 11. It was observed that the lattice design was an effective strategy for reducing the residual stress in the footpad area of the pedal and enabling the additive manufacture of the component through SLM process. This can be attributed to the fact that by increasing the thickness of the pad and introducing porosity inside the pad through lattice structure design, the absorbed heat to volume ratio has decreased, reducing the residual stress effects. Also, increasing the overhang surface of the pad enabled more support structures to prevent part distortion during the SLM process. 
Figure 10 Illustration of internal region of the foot pad after increasing the thickness and filling with lattice structure (see online version for colours)

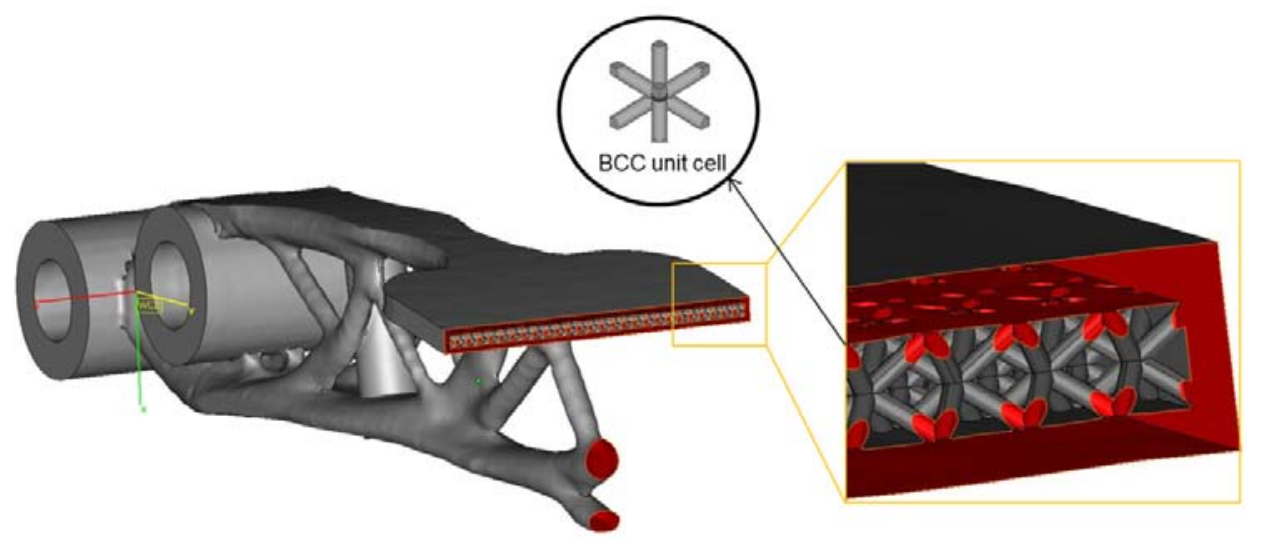

Figure 11 Manufactured topology optimised brake pedal with lattice designed foot pad (see online version for colours)

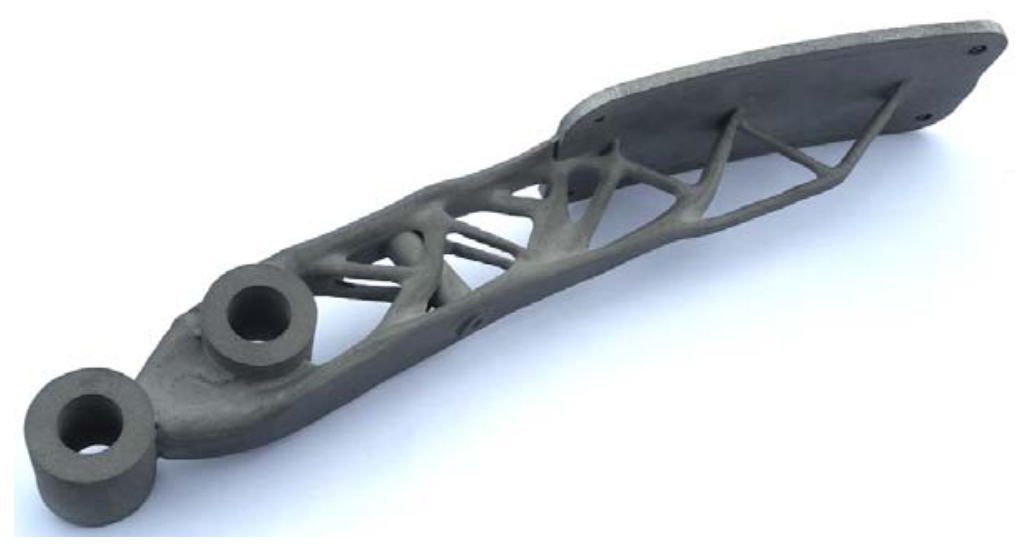

\section{Summary and conclusions}

In this paper, design optimisation of a brake pedal was studied using a recently developed topology optimisation method Iso-XFEM, and lattice structure design. The Iso-XFEM method was found to be capable of optimising real-life structures such as automotive components while the optimisation solutions require only a small amount of postprocessing before sending to additive manufacture. Through the FEA, it was observed that the topology optimisation solutions of the brake pedal had much higher performances compared to the existing pedal. Lattice design was implemented to increase the stability of the foot pad area of the pedal to random loads, and also to reduce the high residual stress induced during the SLM process. It was found that a combination of structural optimisation techniques (e.g., topology optimisation) and intuitive design techniques (e.g., lattice structure design) with an AM process can be used to develop an efficient tool 
for producing energy efficient lightweight components. Future work regarding the application of the method to real powertrain components can include further considerations such as the inclusion of dynamic load cases, thermal effects, frictional behaviours and NVH issues.

\section{Acknowledgements}

This work was supported by the Engineering and Physical Sciences Research Council [grant number EP/I033335/2].

\section{References}

Abdi, M., Ashcroft, I. and Wildman, R. (2014b) 'High resolution topology design with iso-XFEM', in Proceedings of the Solid Freeform Fabrication Symposium, Austin, TX, pp.1288-1303.

Abdi, M., Wildman, R. and Ashcroft, I. (2014a) 'Evolutionary topology optimization using extended finite element method and isolines', Engineering Optimization, Vol. 46, No. 5, pp.628-647.

Aremu, A., Maskery, I., Tuck, C. and Hague, R. (2014) 'A comparative finite element study of cubic unit cells for selective laser melting', in Proceedings of the Solid Freeform Fabrication Symposium, Austin, TX, pp.1238-1249.

Bendsøe, M.P. (1989) 'Optimal shape design as a material distribution problem', Struct. Optim., Vol. 1, No. 4, pp.193-202.

Brackett, D., Ashcroft, I. and Hague, R. (2011) 'Topology optimization for additive manufacturing', in Proceedings of the Solid Freeform Fabrication Symposium, Austin, TX, pp.348-362.

Cooper, D., Thornby, J., Blundell, N., Henrys, R., Williams, M.A. and Gibbons, G. (2015) 'Design and manufacture of high performance hollow engine valves by additive layer manufacturing', Materials and Design, Vol. 69, pp.44-55.

Hsu, Y.L., Hsu, M.S. and Chen, C.T. (2001) 'Interpreting results from topology optimization using density contours', Computers and Structures, Vol. 79, No. 10, pp.1049-1058.

Huang, X. and Xie, M. (2010) Evolutionary Topology Optimization of Continuum Structures: Methods and Applications, John Wiley \& Sons, Chichester, UK.

Hussein, A., Hao, L., Yan, C., Everson, R. and Young, P. (2013) 'Advanced lattice support structures for metal additive manufacturing', Journal of Materials Processing Technology, Vol. 213, No. 7, pp.1019-1026.

Kandreegula, S.K., Sukumar, N., Endugu, S. and Gupta, U. (2015) Structural Non-Linear Topology Optimization of Transmission Housing and Its Experimental Verification, SAE Technical Paper, 2015-01-0098, DOI:10.4271/2015-01-0098.

Maskery, I., Aremu, A.O., Simonelli, M., Tuck, C., Wildman, R.D., Ashcroft, I.A. and Hague, R.J.M. (2015) 'Mechanical properties of Ti-6Al-4V selectively laser melted parts with body-centred-cubic lattices of varying cell size', Experimental Mechanics, Vol. 55, No. 7, pp.1261-1272.

Papadakis, L., Loizou, A., Risse, J. and Schrage, J. (2014) 'Numerical computation of component shape distortion manufactured by selective laser melting', Procedia CIRP, Vol. 18, pp.90-95.

Qian, M., Xu, W., Brandt, M. and Tang, H.P. (2016) 'Additive manufacturing and postprocessing of Ti-6Al-4V for superior mechanical properties', MRS Bulletin, Vol. 41, No. 10, pp.775-783.

Querin, O.M., Steven, G.P. and Xie, Y.M. (1998) 'Evolutionary structural optimisation (ESO) using a bidirectional algorithm', Engineering Computations, Vol. 15, No. 8, pp.1031-1048. 
Sigmund, O. (2001) 'A 99 line topology optimization code written in Matlab', Structural and Multidisciplinary Optimization, Vol. 21, No. 2, pp.120-127.

Simonelli, M. (2014) Microstructure Evolution and Mechanical Properties of Selective Laser Melted Ti-6Al-4V, Doctoral dissertation, Loughborouh University.

Sukumar, N., Chopp, D.L., Moës, N. and Belytschko, T. (2001) 'Modeling holes and inclusions by level sets in the extended finite-element method', Computer Methods in Applied Mechanics and Engineering, Vol. 190, No. 46, pp.6183-6200.

Victoria, M., Marti', P. and Querin, O.M. (2009) 'Topology design of two-dimensional continuum structures using isolines', Computer and Structures, Vol. 87, No. 1, pp.101-109.

Vora, P., Mumtaz, K., Todd, I. and Hopkinson, N. (2015) 'AlSi12 in-situ alloy formation and residual stress reduction using anchorless selective laser melting', Additive Manufacturing, Vol. 7, pp.12-19.

Wu, P., Ma, Q., Luo, Y. and Tao, C. (2016) 'Topology optimization design of automotive engine bracket', Energy and Power Engineering, Vol. 8, No. 4, p.230.

Zhao, Q., Chen, X., Wang, L., Zhu, J., Ma, Z. D. and Lin, Y. (2015) 'Simulation and experimental validation of powertrain mounting bracket design obtained from multi-objective topology optimization', Advances in Mechanical Engineering, Vol. 7, No. 6, pp.1-10.

Zhou, M. and Rozvany, G.I.N. (1991) 'The COG algorithm, part II: topological, geometrical and general shape optimisation’, Comp. Meth. Appl. Mech. Eng., Vol. 89, Nos. 1-3, pp.309-336. 